\title{
Broken Symmetry and Spacetime
}

\author{
David John Baker \\ Department of Philosophy \\ University of Michigan \\ Angell Hall, 435 S. State St. \\ Ann Arbor, MI 48109 \\ djbaker@umich.edu
}

September 11, 2010

\begin{abstract}
The phenomenon of broken spacetime symmetry in the quantum theory of infinite systems seems to point toward an unorthodox ontology. The standard conception of the physical meaning of these symmetries, as changes in coordinates, conflicts with the apparent physical inequivalence of symmetry-related states. I argue that this apparent conflict is an artifact a subtle error in the extant account of physical equivalence due to Halvorson and Clifton.
\end{abstract}

\section{Acknowledgements}

Thanks (as usual) to Gordon Belot, Hans Halvorson and Laura Ruetsche for many valuable comments and conversations. Thanks also to John Earman, Wayne Myrvold and David Wallace for illuminating discussions of symmetry breaking as it relates to Wigner's theorem. Finally, I must recognize three excellent referees, one of whom in particular provided multiple pages of incisive comments. 


\section{Introduction}

Successful spacetime theories, including Newtonian as well as relativistic mechanics, are invariant under a wide range of symmetries. In certain physically contingent situations, these symmetries are broken by the matter content of spacetime. That is, a symmetry transformation that leaves spacetime unchanged fails to preserve the matter content.

This is little bother when the matter in question is classical. In that case it is easy to maintain a standard picture of (global) spacetime symmetries, according to which translating everything in the universe ten feet to my left, or rotating everything clockwise by 90 degrees, makes no physical difference at all. Instead such transformations amount to a change in notation - they change our description of physical reality (in terms of coordinates), but leave the reality itself unchanged.

When the matter content of spacetime is quantum, however, all of this comes into question - or at least it does if we make some plausible assumptions about which physical quantities to include in the content of quantum theory. So we must apparently discard these plausible assumptions, or else alter the classical notion of spacetime symmetries and their nature.

Both horns of this seeming dilemma are deeply unappealing. Fortunately, as I will argue, the dilemma is only seeming. To motivate it we must conjoin plausible physical assumptions with formal assumptions about what is necessary for physical equivalence in quantum theory. These assumptions, which bear on the physical significance of unitarily inequivalent representations, are attractive on the surface but subtly flawed on inspection, and thus deserve to be rejected. The good news is that the seeming dilemma dissolves. The bad news is that we are left without a rigorous, fully general notion of physical equivalence in quantum theory. I will conclude by suggesting where to look for one.

My suggestion will itself hinge on the notion of a symmetry. The apparent dilemma

brings together two separate threads in recent literature. One thread suggests that the existence of a symmetry connecting two solutions, or sets of solutions, of a physical theory is a mark of physical equivalence. This is especially evident for spacetime symmetries, but has been claimed to hold for symmetries in general. The second thread suggests that physical equivalence between the states of two representations in quantum theory is possible only in the presence of unitary equivalence. In the examples central to this paper, a symmetry can exist between representations without a unitary equivalence. The conflict here is obvious; 
the apparent dilemma about spacetime is really just a special case.

I think its resolution, on reflection, is also obvious. The first thread should be pursued at the expense of the second. In infinite quantum theory, as elsewhere, a symmetry should be enough for physical equivalence.

Since the seeming dilemma about spacetime will make clear the problem with existing notions of physical equivalence, let's begin by motivating the dilemma.

\section{Broken rotational symmetry}

Physical theories, like physical and mathematical objects, possess symmetries. A symmetry of a thing is a group of transformations that map the thing to itself, or leave it unchanged. Thus a square drawn in a plane is symmetric under 90-degree rotations - performing such a rotation on the plane gives you an identical square. Similarly Newtonian mechanics is symmetric under all rotations. Whenever I let go of my pen above my table (assuming I'm standing on Earth), Newtonian mechanics predicts that it will drop - no matter which direction the chair, the pen, the Earth and I are oriented. Rotations leave the laws of physics unchanged. ${ }^{1}$

Imagine a Newtonian ball is placed on the "Mexican hat" surface depicted in Figure 1. What ground states (states of lowest energy) are possible for the ball? If we assume the only relevant force is gravity, the ball's total kinetic and potential energy will be lowest when it is stationary at a point of least possible elevation. This will include any point at the bottom of the "brim" of the Mexican hat. Since there are infinitely many such points, infinitely many ground states are available to the ball.

Now suppose the ball assumes one of these ground states. The system of the hat and the ball then fails to be invariant under rotations. ${ }^{2}$ A 90-degree rotation, for example, will fail to map the system onto itself. Instead it is mapped to a system in which the ball has assumed a different ground state. Whenever a ground state fails to be invariant under a symmetry of the laws, we have a case of spontaneous symmetry breaking. Often it is said

\footnotetext{
${ }^{1}$ It is a good - and philosophically loaded - question what this phrase means, but the assumption is often made that all and only transformations which leave "the dynamics" unchanged are symmetries of "the physics." I will adopt this assumption in what follows, in its algebraic form (which requires that symmetries commute with all dynamical automorphisms of the algebra of observables).

${ }^{2}$ Throughout this example I refer only to rotations around the axis that points out the top of the hat.
} 


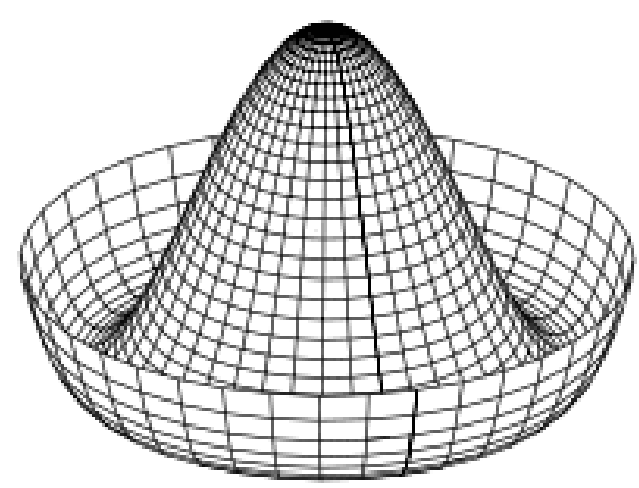

Figure 1: The Mexican Hat

that multiple ground states are available to the system and symmetry transformations map between ground states.

Those familiar with the philosophy of space and time will be hesitant about this last claim, that multiple different ground states are possible for the ball. Of course if the ball and the Mexican hat are sitting on the table in front of me, it makes perfectly good sense to say that the ball could've been closer to me, or farther away. But suppose the ball-hat system is alone in an otherwise empty world (or in a world otherwise symmetric around the hat's axis). Then claiming that there are physically possible worlds in which the ball ends up at a different point along the brim of the hat commits us to some rather strange metaphysics.

What's so strange about this claim? For one thing there is no qualitative difference between a world in which the ball ends up at point $P$ and one in which it's at $P^{\prime}$ (where these are two different points along the brim). One might respond that of course there is a difference - in one world the ball is at $P$, and in the other world it's at a different point $P^{\prime}$. But this assumes some pre-existing means of identifying $P$ and $P^{\prime}$ across worlds. In fact, since $P^{\prime}$ in the second world has all the same qualitative features that $P$ has in the first world (the only qualitative properties that differentiate them are their locations relative to the ball), to suppose these two worlds are different is to suppose that spacetime points possess primitive identity. It must be that some facts other than the qualitative features of a point help determine whether that point is $P$.

Hoefer (1996) and Teller (1991), among others, argue persuasively against the notion that 
spacetime points possess primitive identity. There are two main arguments. First, primitive identity is metaphysically dispensable; everything about points that needs explaining can be explained in terms of their qualitative properties (Hoefer, 1996, 16-22). Second, we have good reason (intuitively, at least) to agree with Leibniz that spacetime symmetry transformations do not relate distinct possible worlds. For example, translating everything in a Newtonian spacetime in the same direction by three feet, or rotating everything by 90 degrees, does not really produce a different state of affairs. These may amount to re-descriptions (in terms of different coordinates) of the same possibility. Or the symmetry may be understood "actively," as rearranging the geometric objects representing physical matter with respect to the geometric structure of spacetime, while denying that such formal rearrangements generate distinct physical situations.

Either way, we are led to a principle according to which global spacetime symmetry transformations never relate different physical possibilities. As Earman and Norton (1987) have shown, denying this principle of "Leibniz equivalence" entails that general relativity is indeterministic. ${ }^{3}$

This line of reasoning leaves us with two attractive ontologies for Newtonian and relativistic spacetime:

Relationism: There are no spacetime points; spatiotemporal properties are nothing more than (actual and possible) relations between physical objects.

Sophisticated Substantivalism: Spacetime points exist, but do not possess primitive identity or haecceity; physical states related by spacetime symmetry transformations describe the same possible world.

Both ontologies entail that in the classical symmetry-breaking case under discussion, there is a unique possible world with least energy, although the state space of our physical theory includes multiple ground states. So there must be a many-to-one correspondence between mathematical states and physical possibilities. Mathematically distinct ground states must

\footnotetext{
${ }^{3}$ Moreover, this indeterminism is of a strange, unobservable sort. Even when initial data determines (as it normally does) the future facts about distance relations between matter fields, it fails to determine which point is occupied by what matter and metrical structure. No probabilistic predictions are available regarding these facts, so the indeterminism is not even "chancy" in the way quantum indeterminism is often taken to be. Leibniz equivalence does away with this indeterminism by denying that different assignments of matter content and metric structure to points give different physical possibilities.
} 
be physically equivalent. More generally, the set of excitations from one ground state must be physically equivalent to the excitations from the other ground states; otherwise there would be modal differences between the ground states, implying that they are not after all physically equivalent.

Plausibly, two sets of states cannot be physically equivalent unless they are intertranslatable in the sense explained by Glymour (1971). There must at least be some way to (bijectively) map between the sets of states and the observable quantities defined on them that preserves (i) the set of physical quantities denoted by the observables and (ii) the values these quantities take on in each state. If this can't be done, it is impossible to claim that the two sets of states denote the same possibilities.

For the ball-hat system, a translation scheme is easy to produce. Suppose the system is described in cylindrical polar coordinates $(r, \theta, h)$. Then we simply perform the coordinate transformation $\theta \rightarrow \theta+\epsilon$ to translate between the ground state in which the ball rests at $\theta=0$ and the one in which it rests at $\theta=\epsilon$. This mapping also takes the set of states describing excitations from the former ground state to the set of excited states for the latter ground state. And it does so in such a way that the values of all classical observables (functions on the space of states) are preserved. The ground states of the ball-hat system thus meet our necessary condition for physical equivalence.

What's the upshot of all this? Mainly that the breaking of spacetime symmetries in classical physics poses no threat to the notion that global spacetime symmetry transformations never correspond to real physical changes. But this notion will come under threat when we consider broken symmetries in quantum physics. Translation schemes, so easy to find in the case of classical symmetries, prove elusive in (infinite) quantum theory.

\section{Representations of quantum theory}

For physical systems with infinitely many degrees of freedom, the possibility of symmetry breaking in quantum theory depends on the existence of inequivalent representations of the theory. So before we explore some examples of quantum symmetry breaking, we would do well to review some basic facts about inequivalent representations. I will do my best to make these facts intelligible for neophytes, so many details will be omitted or relegated to footnotes. 
Inequivalent representations arise when we attempt to quantize the classical theory of an infinite system like a field or a set of infinitely many particles. At large length scales, classical physics provides a good approximation to the more fundamental quantum theory. Quantization is a means of beginning with a classical theory and constructing a corresponding quantum theory that agrees (approximately) with its predictions at large length scales. ${ }^{4}$

Physical quantities in quantum theory are represented by self-adjoint operators, also called observables. These operators act on a collection of state vectors, which must have the mathematical properties of a Hilbert space. For a physically possible state represented by a vector $\psi$ in the Hilbert space, the quantity given by the operator $O$ has its expected value given by

$$
\psi(O)=(\psi, O \psi)
$$

the inner product of $\psi$ with the vector $O \psi$.

In classical mechanics the canonical observables that characterize a state are position and momentum. The first step in quantization is to represent these using self-adjoint operators $q$ (position) and $p$ (momentum). Position and momentum obey the Heisenberg uncertainty relation iff these operators obey the canonical commutation relations (CCRs)

$$
[p, q]=p q-q p=i
$$

So for the vectors of a Hilbert space to represent quantum states, the position and momentum operators on that space must obey (2). We then say that the operators form a representation of the CCRs.

Strictly speaking, a representation of the CCRs cannot act on the entire Hilbert space; this is because the operators $p, q$ are unbounded and so are not well-defined on every vector $\psi$. But a representation of the CCRs in their Weyl form can do better. The exponentiated "Weyl operators" $U(a)=e^{i a q}, V(b)=e^{i b p}$ are well-defined on all $\psi$. So we can ensure that the CCRs hold true for all states by converting them to the Weyl relations,

$$
U(a) V(b)=e^{i a b} V(b) U(a)
$$

\footnotetext{
${ }^{4}$ I will not discuss the measurement problem, which presents a significant challenge to this picture of quantum-classical correspondence.
} 
A representation of the CCRs (in Weyl form) is then a set ${ }^{5}$ of operators on a Hilbert space which obey (3). Quantization then amounts to the construction of such a representation.

Now we're ready to discuss inequivalent representations. In quantum mechanics with finitely many particles (and therefore finitely many degrees of freedom), the Weyl relations uniquely determine the set of all observables. ${ }^{6}$ This makes it possible to suppose that the Weyl relations determine the set of all physical quantities for a finite quantum theory, along with the relations between them. So quantizing a finite classical theory gives a unique result. But this is not true for field theories and statistical mechanics, which require infinite systems. In such cases the Weyl relations do not uniquely determine the complete set of observables on a state space.

Instead, quantizing an infinite system results in a continuum of different Hilbert space representations.(The notion of "same representation" in play here is that of unitary equivalence. ${ }^{7}$ Each representation possesses a different set of observables. Thus, if we suppose that all observables represent physically significant quantities, different representations disagree on which quantities there are. But there is some overlap: the Weyl operators $U(a), V(b)$ generate (via sums and products) a set of operators called the Weyl algebra. ${ }^{8}$ And every representation of the CCRs includes this set of operators. In fact, the Weyl algebra is the largest set of operators shared by all representations.

At first glance, the choice of a representation seems arbitrary. For example, it appears to depend on whether one chooses an accelerating or inertial coordinate system in special relativity (see Halvorson and Clifton, 2001; Arageorgis et al., 2003). A venerable tradition in physics, successful in relativity and gauge field theories, is to suppose that the fundamental physical quantities are invariant under arbitrary choices of perspective. This might motivate us to suppose that the fundamental quantities in quantum theory must be independent of the choice of representation. If so, the fundamental quantities must all correspond to operators

\footnotetext{
${ }^{5}$ More precisely, a pair of one-parameter unitary groups.

${ }^{6}$ Assuming only representations on separable Hilbert spaces are physical, this is established by the Stonevon Neumann theorem.

${ }^{7}$ Two Hilbert space representations $\pi, \chi$ of a $C^{*}$-algebra $\mathfrak{A}$ are unitarily equivalent iff there is a unitary operator $U: \mathcal{H}_{\pi} \rightarrow \mathcal{H}_{\chi}$ such that $U \pi(A) U^{*}=\chi(A)$ for all $A \in \mathfrak{A}$.

${ }^{8}$ This is a $\mathrm{C}^{*}$-algebra: a complex vector space $\mathfrak{A}$ with an associative, distributive product $A B \in \mathfrak{A}$ for $A, B \in \mathfrak{A}$ (i.e. a *-algebra), with a norm $|\cdot|$ obeying $|A B| \leq|A||B|$ and $\left|A^{*} A\right|=|A|^{2}$. A Hilbert space representation of $\mathfrak{A}$ is a mapping $\pi$ from $\mathfrak{A}$ into the bounded operators $\mathcal{B}(\mathcal{H})$ on a Hilbert space $\mathcal{H}$ that preserves the algebraic relations among $\mathfrak{A}$ 's elements.
} 
in the Weyl algebra. Halvorson and Clifton $(2001,425)$ call this position conservatism about observables. $^{9}$

But there is a problem with conservatism, namely that some observables of (seemingly) obvious physical importance are not elements of the Weyl algebra. Ruetsche (2003) focuses on temperature, which is only definable within a representation. Other examples include the stress-energy which is the source of Einstein's gravitational field, and the total number of particles (Ruetsche, 2002, 366-369). If we suppose there could be some matter of fact about the temperature of the universe, or the total number of particles it contains, we must reject conservatism. An obvious alternative is available. Each representation has a unique notion of "closeness" for operators, called its weak topology. We may expand the set of observables by including those which can be approximated arbitrary closely (according to the weak topology) by operators in the Weyl algebra. Halvorson and Clifton have named this approach liberalism about observables. ${ }^{10}$

This is perhaps the most basic dilemma in the interpretation of infinite quantum theory. If we wish to reify quantities like temperature and particle number, which play important roles in physical explanations, we must make a seemingly arbitrary choice of representation in order to define the corresponding observables. Ruetsche $(2002,368)$ has suggested a possible solution: perhaps the choice of representation is not arbitrary after all. According to the Gelfand-Naimark-Segal (GNS) theorem, every quantum state is associated with a unique "home" representation of the Weyl relations. ${ }^{11}$. We may suppose that a state instantiates those physical quantities defined as observables on its home (GNS) representation. On this view, which physical quantities are instantiated is a physically contingent fact, but it is determined by the state. This gives us one way of making liberalism about observables plausible.

The problem with this view - and with any view that entails liberalism about observables

\footnotetext{
${ }^{9}$ Conservatism about observables characterizes the view Ruetsche (2002) calls Algebraic Imperialism.

${ }^{10}$ For Halvorson and Clifton's purposes (and mine), a view will count as liberal about observables if it entails that the physically significant quantities include at least those self-adjoint operators in the double commutant $\pi^{\prime \prime}(A)$ of some representation $\pi(A)$ of the Weyl algebra.

In Ruetsche's parlance, liberalism is (ironically) one tenet of Hilbert Space Conservatism.

${ }^{11}$ The GNS theorem establishes that any abstract state $\omega$ of a $\mathrm{C}^{*}$-algebra $\mathfrak{A}$ is associated with a unique Hilbert space representation $\pi_{\omega}$ of $\mathfrak{A}$ (its GNS representation) in which $\omega$ is represented by a cyclic vector. If $\omega$ is a pure state (as are vacuum or ground states), $\pi_{\omega}$ is irreducible. All the GNS representations that will concern us here are irreducible.
} 
- is that it runs afoul of the standard picture of rotational symmetry discussed in Section 2. In the next section I will explain why.

\section{Quantum symmetry breaking}

To describe the possible outcomes of symmetry breaking in infinite quantum theory (field theory or statistical mechanics), we must appeal to multiple inequivalent representations. To see why, recall from Section 2 that when a symmetry is broken in classical physics there are multiple (mathematically distinct) ground states, related by symmetry transformations. This will not always be the case in infinite quantum physics, since some field-theoretic systems lack a well-defined ground state. But it does hold true in some paradigm examples, which we can use as a guide.

In quantum field theory, the role of a ground state is played by a state called the vacuum (when it exists). A given Hilbert space representation includes (at most) a single vacuum state. So for SSB to occur in such a vacuum representation, ground states in multiple inequivalent representations must be possible.

To see how this works, we must examine how symmetries are represented in quantum theory. In its most general form, a symmetry is given by a transformation (automorphism) of the Weyl algebra. A spacetime symmetry, for example, acts on the coordinates $x, p$. A transformation which takes $x$ to $T(x)$ then takes $U(a)=e^{i a x}$ to $T(U(a))=e^{i a T(x)}$; likewise for momentum and $V(b)$.

Symmetries must of course preserve expectation values, or else we could distinguish empirically between transformed and un-transformed systems. To meet this condition a symmetry must also include a mapping $T^{\prime}$ on states, taking the state $\psi$ to $T^{\prime}(\psi)$. In general for states $\psi$, observables $O$ and symmetry transformations $T$, we require that $T^{\prime}(\psi(T(O)))=$ $\psi(O) .{ }^{12}$

Consider a ground state $\psi_{0}$ in its home (GNS) representation. If the ground state is invariant under a symmetry $T$ - i.e., if $T^{\prime}\left(\psi_{0}(O)\right)=\psi_{0}(O)$ for all $O$ - then $T$ acts as a unitary operator on the GNS representation. ${ }^{13}$ But if $T$ is a broken symmetry, ground states are not

\footnotetext{
${ }^{12}$ Since $T$ is an automorphism of the algebra of observables and a state can be considered a normed linear functional on the same algebra, this requirement entails that $T^{\prime}(\psi)=\psi \circ T^{-1}$.

${ }^{13}$ For purposes of this essay I do not distinguish between unitary and antiunitary operators. Note that if transformation $T^{\prime}(\psi)$ is given by a unitary operator $U \psi$, then the transformed operator $T(O)$ is given by
} 
invariant under $T^{\prime}$, so nothing guarantees that $T$ will act like a unitary operator. In fact, whenever a symmetry of a quantum system is broken, $T$ will fail to act like a unitary operator - the symmetry will not be unitarily implementable. This has been taken, for instance by Earman (2004) and Strocchi (2008), to be the defining feature of quantum symmetry breaking. Since $T$ is not unitary, it maps between unitarily inequivalent representations. This is why inequivalent representations are required for the quantum description of symmetry breaking.

Are Earman and Strocchi correct to define SSB as the existence of a symmetry which is not unitarily implementable? For our purposes what matters is not whether their definition is correct, nor even the generally agreed-upon claim that non-unitary symmetry is a necessary condition for SSB. All that's needed to motivate the dilemma to come is the well-established fact that some physically relevant examples of SSB involve rotational symmetries which cannot be unitarily implemented. As long as one such example can be found, it can be employed to motivate the dilemma.

We already discussed broken rotational symmetry in classical physics. This sometimes occurs in infinite quantum theory as well. One example occurs in the case of coherent states which arise in the so-called infrared problem in quantum field theory (see Roepstorff, 1970). Though I expect this example will be unfamiliar to most readers, it is formally simple. To construct a coherent state with broken rotational symmetry, we begin with a rotationally invariant state $\psi_{0}$. In field theory, there are infinitely many configuration and momentum observables $q_{i}, p_{j}$ which obey the CCRs (2) for $i=j$. From these we can construct Weyl operators $U_{i}(a), V_{j}(b)$ obeying $(3)$. $\psi_{0}$ then assigns expectation value $\psi_{0}(W)$ to each Weyl operator $W$.

A transformation which takes $\psi_{0}$ to another state that also obeys the CCRs is called a canonical transformation. Among these are the coherent transformations, which take the momentum expectation values $\psi\left(p_{j}\right)$ to $\psi\left(p_{j}\right)+\lambda_{j}$, for given scalar $\lambda_{j}$ 's. In terms of the Weyl operators, a coherent transformation takes $V_{j}(b)$ to $e^{i \lambda_{j}} V_{j}(b)$. Call the transformed state $\psi_{\lambda}$. Like all states, $\psi_{\lambda}$ has a "home" GNS representation. $\psi_{\lambda}$ 's home representation is unitarily equivalent to $\psi_{0}$ 's iff

$$
\sum_{j}\left|\lambda_{j}\right|^{2}<\infty
$$

$U^{*}$. 
For some choices of $\lambda_{j}$, applying a coherent transformation to $\psi_{0}$ will give us a state with broken rotational symmetry. To see how this happens, note first that symmetries are most generally given by transformations (automorphisms) on the Weyl algebra. Thus a rotation $R$ transforms the Weyl operators $V_{j}(b)$ to $R\left(V_{j}(b)\right)$. For a coherent state $\psi_{\lambda}$, this means that $R$ transforms $V_{j}(b)+\lambda_{j}$ to $R\left(V_{j}(b)\right)+R\left(\lambda_{j}\right)$. Now suppose that the $\lambda_{j}$ are chosen so that

$$
\sum_{j}\left|\lambda_{j}-R\left(\lambda_{j}\right)\right|^{2}=\infty
$$

Then $\psi_{\lambda}$ and the rotated state $R\left(\psi_{\lambda}\right)$ have inequivalent GNS representations. Rotational symmetry transformations take $\psi_{\lambda}$ outside its home representation; thus, the rotational symmetry is broken for such a state. ${ }^{14}$

A mathematically more difficult but physically more familiar case is that of ferromagnetism, detailed in Ruetsche (2006). Here we must work with representations of the canonical anticommutation relations (CARs). ${ }^{15}$ The main payoff is just this: suppose $\psi_{M}$ is the ground state of an infinitely long bar magnet. In such a case the magnet will be polarized in the direction of its North pole. Then the rotated state $R\left(\psi_{M}\right)$ is polarized along a different axis and lives in a different, unitarily inequivalent representation from $\psi_{M}$. No unitary operator can map between the set of states reachable by exciting the ground state $\psi_{M}$ and those reachable from $R\left(\psi_{M}\right)$. Again rotational symmetry is broken.

The infinite magnet model has some disadvantages as a basis for the argument of this paper. For one thing, it is highly idealized as a description of realistic systems (though I would maintain that infinite magnets are physically possible, whether actual or not). It is also not clear that the spontaneously broken symmetry in such examples is always the group of global rotations. ${ }^{16}$ But the magnet case is intuitive, so I will employ it in the following

\footnotetext{
${ }^{14} \mathrm{~A}$ definitional ambiguity here is worth noting. Coherent representations do not meet my informal definition of SSB - the existence of a non-invariant ground state - since coherent representations lack ground states. But they do meet Earman and Strocchi's mathematical definition of SSB in QFT, since the rotational symmetry is not unitarily implementable. The latter sort of "symmetry breaking," the existence of a non-unitarily implementable symmetry, is what's needed as a premise for the liberal's dilemma, while non-invariant ground states are not needed. So since coherent states exhibit the mathematical features I need to get my argument started, I will lump them under the moniker of SSB although they lack one of its physically intuitive features.

${ }^{15}$ The extra mathematical difficulty arises from the fact that the relevant Weyl algebra for the CARs is not the same as the one I present above, which applies only to the CCRs.

${ }^{16}$ In the idealized Heisenberg model Ruetsche (2006) discusses, the magnet is modeled as a fixed lattice
} 
discussion. The skeptical reader should keep in mind that I could always substitute the more straightforward example of coherent states instead of relying on the infinite magnet.

\section{$5 \quad$ Intertranslatability of representations}

We saw at the end of Section 2 that two states, or more generally two sets of possible states, can only be physically equivalent if they are intertranslatable. Since representations of a quantum theory come with associated sets of states (folia), we would like a notion of intertranslatability for quantum theory that tells us when a translation scheme exists between two sets of states. But existing notions of intertranslatability, including the one we will employ due to Glymour (1971), mostly discuss intertranslatability of theories.

Fortunately, I think a necessary condition for sets of states to be intertranslatable can be defined if we have a prior notion of intertranslatability for theories. Consider two sets of states $S, S^{\prime}$. For each of these two sets we can define a toy theory according to which that set gives an exhaustive list of the physically possible states. For example, we may call $T_{S}$ the theory according to which all and only states in $S$ are physically possible. ${ }^{17}$ I propose that $S$ and $S^{\prime}$ are intertranslatable only if there is a translation scheme between the theories $T_{S}$ and $T_{S^{\prime}}$. Imagining that a hypothetical character believes that the physically possible states are just the states in $S$, and his brother claims the possibilities are given by the states in $S^{\prime}$, it seems we should deny that $S$ and $S^{\prime}$ are intertranslatable if the two brothers cannot find a means of translating between their theories according to which their predictions agree.

This account of intertranslatability for sets of states allows us to determine whether representations in quantum theory are intertranslatable without presuming (objectionably) that a representation corresponds in any way to a "theory." It will also permit an answer to the following pressing question. Take a state with rotationally broken symmetry like our

with a spin-1/2 particle at every point. This means that not all global rotations can be performed on the system as described. The broken symmetry in this case is not the group of all global rotations, but rather the synchronized rotations of every spin at its fixed lattice point. But for some special cases (e.g. 90 degree rotations of the three-dimensional lattice, or 180-degree rotations of the one-dimensional lattice) these spin rotations coincide with global rotations. So even in this simplified model, some (though not all) global rotations are spontaneously broken in a rigorous sense.

${ }^{17}$ Constructing $T_{S}$ will require a specification of which observable magnitudes are physically significant. In the examples we'll discuss, this is determined by whether we assume the point of view of the liberal or the conservative about observables. 
infinitely long magnet $\psi_{M}$. Could the state $\psi_{M}$ be physically equivalent to the rotated state $R\left(\psi_{M}\right)$ ? Moreover, could the set of excited states reachable from $\psi_{M}$ be physically equivalent to the states reachable from $R\left(\psi_{M}\right)$ ?

Before we answer these questions, let's try to see what's at stake. Recall the two most plausible ontologies for spacetime, relationism and (sophisticated) substantivalism. Both entail that spacetime symmetry transformations do not represent real physical changes in a system. This means a physically possible world is, on these views, given by an equivalence class of solutions, according to which any two solutions related by a symmetry are (physically) equivalent. If we generalize this principle to sets of solutions, it likewise follows that if a particular symmetry transformation gives a bijection between two such sets, these sets should be physically equivalent. All of this appears straightforwardly inconsistent with the possibility that a spacetime symmetry transformation - for instance, a global rotation could relate two physically inequivalent states or sets of states.

This means that if the set of possibilities in which our magnet is polarized along the $-x$ direction is physically inequivalent to the set in which it's polarized along $+x$, both sophisticated substantivalism and relationism are in trouble. So we might hope that sensible ontologies for quantum theory, including liberalism about observables, would at least permit us to translate between the $-x$-polarized states and the $+x$ states. But when rotational symmetry is broken, liberalism about observables seems not to permit this.

This is a consequence of the unitary inequivalence between the $-x$ ground state's GNS representation and the $+x$ representation. A theorem of Halvorson and Clifton (2001, Prop. 4) entails that

- Assuming liberalism about observables, there is a translation scheme between two (irreducible) representations only if the representations are unitarily equivalent. ${ }^{18}$

Unless some metaphysical fix can be found, this theorem seems to be saying that Leibniz equivalence, the basis for our (arguably) best ontologies of spacetime, is incompatible with liberalism.

\footnotetext{
${ }^{18}$ More precisely, Halvorson and Clifton show that a factor representation and and irreducible representation are intertranslatable only if they are quasi-equivalent. In the case where both representations are irreducible, quasi-equivalent representations are unitarily equivalent. In our key examples (coherent states and ferromagnets), the relevant representations are GNS representations of pure states, hence irreducible, so we may ignore this distinction.
} 
Indeed, a much weaker thesis than Leibniz equivalence also appears to be ruled out. Leibniz equivalence entails that no spacetime symmetry transformation can relate physically inequivalent (sets of) states. The theorem of Halvorson and Clifton indicates that no spontaneously broken symmetry transformation can relate physically equivalent representations.

This result is incompatible with the much weaker (compared with Leibniz equivalence) thesis that some spontaneously broken symmetry transformations can relate physically equivalent states. So the liberal who denies Leibniz equivalence but wants to accept that (for example) at least some rotations of an infinite magnet correspond to mere changes of coordinates is also under threat.

\section{Metaphysical fixes}

A similar problem for relationism and sophisticated substantivalism (call the disjunction of these views the standard picture) has arisen before. It was prompted by the case of parity asymmetry in the standard model. In this case a fix was found; perhaps it can be extended to cover the present case.

The earlier problem arose from the fact that the gauge theory describing the weak nuclear interaction is not invariant under parity transformations. Parity, or mirror reflection across one spatial axis, is a transformation which changes the handedness (or chirality) of structures like hands which possess incongruent counterparts. Some quantum fields, including the Dirac electron, possess this sort of handedness. And the gauge bosons ("force-carrying" fields) of the weak interaction couple only with left-handed, as opposed to right-handed, Dirac fields.

This is curious when we reflect for a moment on what a parity transformation amounts to. To invert parity, we pick a spatial axis ( $x$, say) and an origin; then we reverse the coordinates of each point along that axis (taking $x$ to $-x$ ). This is nothing but a change of coordinates, so the standard picture ought to entail that no two worlds related by a parity transformation are distinct possibilities. But here are two such worlds: one in which the weak bosons couple with right-handed Dirac fields, and one in which the bosons couple with left-handed Dirac fields. Weak theory tells us that while the first world is physically impossible, the second is possible. Since the same world can't be both possible and impossible, parity transformations must sometimes relate distinct worlds.

For a fix to work, some mistake must be found in the above reasoning. The project of 
rectifying this mistake was completed in stages by Hoefer (2000), Huggett (2000) and Pooley (2003). Briefly, the solution is as follows. For the above reasoning to be correct, there must be some intrinsic difference between right- and left-handed Dirac fields. But the handedness of fields is defined only in relation to a conventional, right-handed coordinate system (one in which we use the "right-hand rule" to compute cross products). In particular, the theory predicts no difference between right-handed fields written in right-handed coordinates and left-handed fields written in left-handed coordinates.

This means that, while it is physically necessary in weak theory that there be a preferred handedness, it is a matter of convention whether that handedness is right or left. The theory asserts merely that there exists a preferred handedness, not which handedness it is. We can thus maintain, in accordance with the standard picture, that there is no matter of fact about "which" handedness is preferred. So, while the theory entails a physical difference between electrons of the preferred handedness and electrons with the opposite handedness, it does not entail a difference between right- and left-handed electrons, because there is no matter of fact about whether the preferred handedness is right or left. In a world with a single electron, there will be no fact of the matter about whether the electron is right or left handed, but it will be determinate whether the electron has a handedness which allows it to couple with the weak bosons.

Let's see if a similar fix can apply to our example of a quantum magnet with broken rotational symmetry. Our dilemma, remember, is that the theory seems to predict a physical difference between the possible magnets polarized along the $-x$ direction and those polarized along $+x$. But, analogous to the parity case, these claims are being made within a coordinate system in which we've conventionally "painted on" $x$ and $z$ axes. These names for the axes are pure convention, and could have been reversed without changing the physics. So we may conclude, as in the parity case, that the physics is not telling us about any intrinsic difference between $+x$ magnets and $-x$ magnets. Rather, it is telling us that some direction is in fact preferred, and there is a physical difference between systems oriented along that direction and systems oriented along a different direction. But none of this entails that coordinate changes represent real physical changes.

This fix would work perfectly well if the magnet case were properly analogous to the weak theory. But it isn't. The existence of a preferred direction in weak theory is ensured by the parity-asymmetry of the laws. But in the case of our magnet, the rotational symmetry 
of the laws should similarly ensure the nonexistence of a preferred direction.

To deny this is to deny any substantive difference between spontaneously broken symmetry and asymmetry. In cases of broken rotational symmetry, the laws still entail the nonexistence of a preferred direction, so any metaphysical fix appealing to such a direction is in violation of the laws. The problem for liberalism is not just that it requires solutions related by rotations to be physically distinct. It requires that they be distinct solutions to a theory that is symmetric under rotations. We cannot solve the problem by denying that rotation is a symmetry of the theory, which we must do in order to apply the parity-fix.

\section{Deeper problems}

It's beginning to appear as if there's no way out except to deny either liberalism or the standard picture of spacetime symmetries. But I'm not about to ask you to choose. This appearance of inevitability is an artifact of the way I've presented the problem so far.

It's time to broaden the scope of our inquiry beyond the carefully chosen question of whether the $-x$-polarized states of a magnet are, for the liberal about observables, physically equivalent to the $+x$-polarized states. To make sure that denying liberalism will get us out of my dilemma, we must pose the same question to the conservative about observables (who believes that the only physically significant observables live in the Weyl algebra). And this is where things become truly strange. The conservative's answer is no different from the liberal's.

This fact was established by Halvorson and Clifton (2001), but the reason why isn't obvious from their presentation. The important result for the conservative about observables is the following:

- On the "conservative approach to states," there is a translation scheme between two (irreducible) representations only if they are unitarily equivalent. (Halvorson and Clifton, 2001, Prop. 3)

A conservative-about-states is supposed to hold that the density operators of one of the two representations (the representation's folium) gives an exhaustive list of the physically possible states. Nothing about conservatism about observables entails conservatism-about-states, so why does this result matter? 
The answer has to do with how Clifton and Halvorson are thinking of translation. They view it as a relationship between complete (exhaustive) sets of physically possible states. This means that, in establishing that two representations are intertranslatable for the conservative-about-states only if unitarily equivalent, they've also established something else. Their result implies that the folia of two representations are intertranslatable only if the representations are unitarily equivalent. And this must be true, independent of whether one actually is a conservative-about-states.

This can be seen by employing my condition (from Section 5) on intertranslatability for sets of states. Two folia are intertranslatable only if the two hypothetical theories according to which one folium or the other includes all possible states are intertranslatable. These hypothetical theories are just the theories of two conservatives-about-states who count the two folia as physically possible. So even someone "liberal" about states, who counts many more states as physically possible, must still agree that the sets of states counted as possible by two conservatives-about-states are intertranslatable only if they're unitarily equivalent. It's just that he disagrees with the conservatives about whether these states are the only possible ones.

Nothing about the above reasoning presumes liberalism about observables. On the assumptions of Halvorson and Clifton, it is therefore true, even for the conservative about observables, that the states of two disjoint folia are physically inequivalent. Since the $-x$ polarized states of a magnet form a folium disjoint from its $+x$-polarized states, even the conservative must agree that these two sets of possibilities are not physically equivalent.

I imagine you'll agree that it's time to examine the assumptions of Halvorson and Clifton. Glymour's notion of translation between theories is their starting point. This requires that any translation map the primitive terms (fundamental quantities, in physics) of one theory to those of the other, so that the theorems (predictions) of the two theories are logically equivalent. So to translate between the toy 'theories' given by two representations, Halvorson and Clifton must identify the primitives and theorems of a given quantum theory. Finding the theorems is easy; these are just the predictions each state makes about the values of the observables. So Clifton and Halvorson require that any translation scheme include bijective mappings $\beta$ between the states of the two toy 'theories' and $\alpha$ between 
their observables, such that, for all observables $A$ and states $\omega$,

$$
\beta(\omega)(\alpha(A))=\omega(A) .
$$

(Note that which operators $A$ count as observables will depend on whether you're a conservative or liberal about observables.) This strikes me as a straightforward and reasonable requirement for any translation scheme.

Their other requirement is more suspect. They suggest:

Think of the Weyl operators $[U(a), V(b)]$ as the primitives of our two 'theories,' in analogy with the way the natural numbers can be regarded as the primitives of a 'theory' of real numbers. (Halvorson and Clifton, 2001, 429)

The analogy is that all observables can be constructed, either by algebraic operations or taking limits, from Weyl operators and series of Weyl operators. They then require that, for all such "primitive" Weyl operators $W$,

$$
\alpha\left(\pi_{1}(W)\right)=\pi_{2}(W) .
$$

This is the condition responsible for their Proposition 3, that for the conservative-aboutstates two representations are intertranslatable only if unitarily equivalent. It requires that any translation take the concrete unitary operator representing each Weyl operator in representation $\pi_{1}$ to the one representing the very same Weyl operator in $\pi_{2}$. In particular, it rules out the possibility that the representative some Weyl operator in $\pi_{1}$ should be mapped in translation to a different Weyl operator's representative in $\pi_{2}$.

This is violated even by unitary operators which implement non-trivial symmetry transformations, since symmetries often take one Weyl operator to a different one which is (for instance) localized in a different region of spacetime. Sometimes a symmetry can guarantee the existence of a translation scheme in Halvorson and Clifton's sense - but only if the symmetry is unitarily implementable. In general, a symmetry between representations does guarantee the existence of an automorphism $T: \mathfrak{A} \rightarrow \mathfrak{A}$ such that

$$
\alpha\left(\pi_{1}(W)\right)=\pi_{2}(T(W)) .
$$

But this can hold true without implying (7), and moreover it does not suffice to prove 
Halvorson and Clifton's proposition. Even if the Weyl operators do deserve to be treated as primitive, I see no physical motivation for implementing this requirement by holding translation schemes to criterion (7) rather than (8).

There are many reasons to question requirement (7). Immediately, one worries that Halvorson and Clifton may be equivocating in their use of 'primitive,' when justifying the Weyl operators as the primitive terms in quantum theory. Is the sense in which a physical theory's basic terms are primitive really the same as the sense in which the natural numbers are primitive in number theory?

This general worry is supported by some concrete concerns about their specific choice of primitives. First: why shouldn't all observables count as primitives? Second: why must our two theories agree about the correspondence between the mathematical Weyl operators and the primitive physical quantities? Together, the criteria $(6,7)$ require that a translation map every state to one that agrees with it on the expectation values of all Weyl operators. So a state that assigns the Weyl operator $W_{1}$ the expectation value 1 and $W_{2}$ the value 0.5 must be mapped to another state that assigns 1 to $W_{1}$ and 0.5 to $W_{2}$. But why couldn't the mathematical object $W_{1}$ denote a different physical quantity in different representations? If it could, we should allow a translation scheme to map our hypothetical state to one in which $W_{1}$ has expectation value 0.5 and $W_{2}$ 's expected value is 1 .

This is the sort of "translating" that symmetry transformations do all the time. For example, when we take a model of a $+x$-polarized magnet and transform the $x$ coordinate to $-x$, we will change the expectation value of the observable for magnetization along $x$, say from 1 to -1 . The believer in Leibniz equivalence would say that we have thereby changed which physical quantity this operator denotes. As long as we keep that in mind, there should be no obstacle to constructing a translation scheme.

What this example shows, I think, is that the Halvorson-Clifton criteria themselves contradict Leibniz equivalence. So it should be no surprise that they lead to a dilemma for the liberal (or anyone else) who wants to accept Leibniz equivalence.

Let's step back and look at the dialectic. We've seen that the Halvorson-Clifton translation criteria $(6,7)$ by themselves are enough to generate the problem raised in section 5 for both the liberal and the conservative about observables. Together with the physical possibility of broken spacetime symmetry, these criteria entail that states related by global rotations of everything in the universe can fail to be physically equivalent. This result is in serious 
tension with the best available metaphysics for spacetime. We've also seen that independent concerns arise, to the effect that the Halvorson-Clifton criteria may be too stringent. Some response may be possible, on behalf of their criteria, but in light of the serious dilemma for the standard picture of spacetime that results from accepting the criteria, I think the best option is clearly to abandon them.

I should note that Halvorson now agrees with me, writing in a recent erratum that

The original definition of physical equivalence assumes that the two observers already have established a translation scheme between their primitives i.e. $\left[\pi_{1}(A)\right]$ corresponds to $\left[\pi_{2}(A)\right]$. But this requirement is overly restrictive, because once the $\left[\pi_{1}(A) \rightarrow \pi_{2}(A)\right]$ correspondence is fixed, the translation scheme between states is also fixed (i.e. the two theories are intertranslatable only if they have the same states relative to the fixed correspondence...

In other words, their criteria placed too strong a restriction on the admissible translation schemes, effectively limiting them to a single method. That said, their use in Halvorson and Clifton (2001) was restricted to the section on physical inequivalence of representations, and does not affect that paper's other arguments about the observer-dependence (and thus the nonexistence) of particles in QFT.

\section{Rethinking intertranslatability}

In an important sense, our philosophical picture of QFT is left adrift by this new result. We are left without a working definition of intertranslatability. The Halvorson-Clifton criteria fall short when we look at cases of broken spacetime symmetry.

Alternative criteria for intertranslatability of representations will have to be found. One sufficient condition, it seems to me, is the existence of a symmetry mapping between representations. One purpose of this paper was to pose and deal with apparent counterexamples to this sufficient condition. ${ }^{19}$ My suggestion has a corollary: since symmetries can fail to

\footnotetext{
${ }^{19} \mathrm{My}$ views here should be carefully distinguished from the mistaken notion that any $\mathrm{C}^{*}$-algebraic automorphism is sufficient for physical equivalence. This would, for example, make the coherent representations of Section 4 physically equivalent to the Fock representation, which they obviously are not. Not every automorphism of a $\mathrm{C}^{*}$-algebra is a symmetry - in order to be one, it must also commute with the dynamics.
} 
be unitarily implementable, it implies that unitary equivalence is not necessary for physical equivalence, contrary to the claims of (for example) Arageorgis $(1995,302) .{ }^{20}$

What of the liberal's seeming dilemma? It appeared at the end of Section 7 to be a pseudo-problem, a special case of a misunderstanding that threatened every interpreter of infinite quantum theory. But while we have witnessed the demolition of a supposed proof that the liberal cannot always treat symmetry-related possibilities as physically equivalent, there is no proof that the liberal can. And there are reasons - imprecise but suggestive reasons - to think she can't.

The fact remains that a symmetry is defined as an automorphism of a $\mathrm{C}^{*}$-algebra (Weyl algebra) which includes only a proper subset of the observables the liberal counts as physically significant. This definition entails that the expectation values of Weyl observables are preserved by the symmetry. There is no similar entailment for the extra observables recognized by the liberal unless the symmetry is unbroken (unitarily implementable). So while the conservative who recognizes only Weyl observables is on safe ground accepting symmetry as a mark of physical equivalence, the liberal who accepts this is going out on a limb.

The resolution of the liberal's dilemma will depend on a new definition of intertranslatability, something I can't provide here. But I hope the urgent need for a new definition has at least been made clear. We don't yet know what physical equivalence and intertranslatability are in infinite quantum theory, but we now know more about what they can't be - and that is progress.

\section{References}

Arageorgis, Aristidis (1995), Fields, Particles and Curvature, University of Pittsburgh: unpublished dissertation.

Arageorgis, Aristidis, John Earman and Laura Ruetsche (2003), "Fulling Non-uniqueness and the

\footnotetext{
${ }^{20}$ Arageorgis justifies his position by appeal to Wigner's theorem, which establishes that any mapping between Hilbert spaces which preserves the transition probabilities of vector states must be unitary. Arageorgis $(1995,302 \mathrm{fn} 111)$ claims that this implies two representations can only agree about transition probabilities if they're unitarily equivalent. This step in his argument rests on a confusion: the nonexistence of the intertwining operator required for unitary equivalence does not imply that there is no unitary mapping between the vector states of the representations in question. In fact, as Roberts and Roepstorff (1969, Prop. 6.1) prove, any algebraic symmetry preserves all transition probabilities.
} 
Unruh Effect: A Primer on Some Aspects of Quantum Field Theory," Philosophy of Science 70:164-202.

Earman, John (2004), "Curie's Principle and spontaneous symmetry breaking," International Studies in the Philosophy of Science 18:173-198.

Earman, John and John Norton (1987), "What Price Spacetime Substantivalism? The Hole Story," British Journal for the Philosophy of Science 38:515-525.

Glymour, Clark (1971), "Theoretical Realism and Theoretical Equivalence," in Boston Studies in Philosophy of Science VIII, Reidel: Dordrecht, 275-288.

Halvorson, Hans and Rob Clifton (2001), "Are Rindler quanta real? Inequivalent particle concepts in quantum field theory," British Journal for the Philosophy of Science 52:417-470.

Hoefer, Carl (1996), "The Metaphysics of Spacetime Substantivalism," Journal of Philosophy 93:527.

Hoefer, Carl (2000), "Kant's hands and Earman's pions: chirality arguments for substantival space," International Studies in the Philosophy of Science 14:237-256.

Huggett, Nick (2000), "Reflections on parity non-conservation," Philosophy of Science 67:219-241.

Pooley, Oliver (2003), "Handedness, parity violation, and the reality of space," in Brading, Katherine and Elena Castellani (eds.), Symmetries in Physics: Philosophical Reflections, Cambridge: Cambridge UP, 250-280.

Roberts, John E. and Gert Roepstorff (1969), "Some Basic Concepts of Algebraic Quantum Theory," Communications in Mathematical Physics 11:321-338.

Roepstorff, Gert (1970), "Coherent Photon States and Spectral Condition," Communications in Mathematical Physics 19:301-314.

Ruetsche, Laura (2002), "Interpreting Quantum Field Theory," Philosophy of Science 69:348-378.

Ruetsche, Laura (2003), "A Matter of Degree: Putting Unitary Inequivalence to Work," Philosophy of Science (Proceedings) 70:1329-1342.

Ruetsche, Laura (2006), "Johnny's So Long at the Ferromagnet," Philosophy of Science (Proceedings) 73:473-486.

Strocchi, Franco (2008), Symmetry Breaking, Berlin/Heidelberg: Springer.

Teller, Paul (1991), "Substance, Relations and Arguments about the Nature of Space-Time," Philosophical Review 100:363-397. 